Reprod. Nutr. Dévelop., 1988, 28 Suppl. n¹, 165-166

\title{
Variations nycthémérales de la concentration en ammoniac dans le liquide du rumen, chez les vaches laitières dans la période péri-partum
}

D. DEHARENG, J.-M. GODEAU

Laboratoire de Biochimie Normale et Pathologique, Faculté de Médecine Vétérinaire,

Rue des Vétérinaires, 45 à B-1070 Bruxelles, Belgique.

Summary. Automated and continuous recording of ammonia concentration in rumen liquor (Godeau et al., 1986) was performed on four cows around their first calving. Results suggest an unsufficient concentration in early lactation.

Les variations nycthémérales du $\mathrm{NH}_{3}$ du liquide du rumen de vaches laitières sont étudiées en période péri-partum au moyen de la technique en continu décrite par Godeau et al. (1986).

Matériel et méthodes. Quatre primipares $(17,5 \mathrm{~kg}$ lait/jour) canulées dans le rumen reçoivent leurs rations en 2 repas journaliers égaux (6 h 15-17 h 30 ). L'ingestion d' $1 \mathrm{~kg}$ de foin est suivie de la distribution du complément de la ration dont la constitution n'a varié qu'après le part et au $2^{\mathrm{e}}$ jour des semaines $+1,+3$ et +5 post-partum (tabl. 1 ). Au cours des semaines $-10,-3,-2,+2$ à +6 et +8 , le dosage nycthéméral en continu ( $24 \mathrm{~h} /$ animal testé) du $\mathrm{NH}_{3}$ du liquide du rumen (Godeau et al., 1986) permet d'y délimiter (fig. 1) les pics ammoniacaux postprandiaux qui correspondent à l'évolution du $\mathrm{NH}_{3}$ exogène. Pour étudier la dégradation de l'azote $(\mathrm{N})$ alimentaire en période péri-partum, les surfaces observées sous la courbe (calculées par sommation des concentrations mesurées à chaque minute) des 2 pics post-prandiaux journaliers sont comparées aux surfaces théoriques qui sont attendues au départ (a) : du $\mathrm{N}$ dégradable (Alimentation des Ruminants, I.N.R.A. 1980) et de la matière organique digestible ingérés, ou (b) : de l'évolution des surfaces en fonction du $\mathrm{N}$ ingéré, les surfaces observées en semaine -3 étant prises comme référence dans les 2 cas.

Résultats et discussion. Les valeurs observées du pic de $\mathrm{NH}_{3}$ augmentent comme les valeurs attendues (tabl. 2) en fonction de l'accroissement des quantités d'azote total ou dégradable ingérées, avec l'avancement de la lactation. Elles leur sont toutefois inférieures en $2^{e}$ et $3^{e}$ semaines, ce qui suggèrerait un certain retard dans l'adaptation de la population microbienne. La durée journalière pendant laquelle la concentration en $\mathrm{NH}_{3}$ est insuffisante $(50 \mathrm{mg} / \mathrm{l}$ selon Pisulewski et al., 1981) pour les bactéries, diminue assez régulièrement quand l'azote dégradable ingéré augmente en quantité ou en concentration dans la matière organique digestible. 

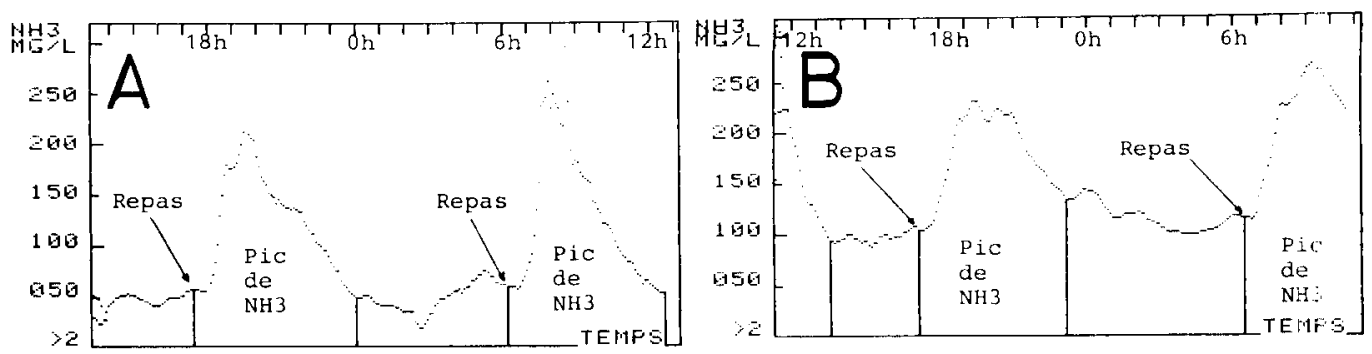

FIG. 1. - Exemples d'évolution des teneurs en $\mathrm{NH}_{3}$ du liquide du rumen chez deux vaches différentes (A: semaine $+3 ;$ B : semaine +8 ).

TABL. 1. - Constitution de la ration $(\mathrm{kg} / \mathrm{j})$.

\begin{tabular}{lccccc}
\hline Semaines péri-partum & -10 à -2 & +1 et +2 & +3 et +4 & +5 & +6 à +8 \\
\hline Foin de prairie & 3,25 & 3,50 & 4,00 & 4,00 & 4,00 \\
Pulpes sèches de betterave & 1,50 & 1,00 & 1,00 & 1,00 & 1,00 \\
Orge aplatie & 1,40 & 1,00 & 1,00 & 1,00 & 1,00 \\
Concentré du commerce & 4,00 & 4,50 & 5,50 & 5,00 & 5,00 \\
Tourteau de soja & 0,00 & 0,80 & 1,00 & 2,00 & 2,00 \\
\hline
\end{tabular}

TABL. 2. - Evolution des quantités ingérées et des paramètres relatifs aux profils nycthéméraux de l'ammoniaque.

\begin{tabular}{|c|c|c|c|c|c|c|c|c|c|}
\hline Semaines péri-partum & -10 & -3 & -2 & +2 & +3 & +4 & +5 & +6 & +8 \\
\hline $\begin{array}{l}\text { Quantités ingérées: } \\
\text { matière sèche }(\mathrm{kg} / \mathrm{i})\end{array}$ & 9,0 & 9,0 & 9,0 & 9,8 & 11,4 & 11,4 & 12,0 & 12,1 & 12,1 \\
\hline matière organique digestible & 6,3 & 5,9 & 5,8 & 6,2 & 7,7 & 7,2 & 7,9 & 8,3 & 8,4 \\
\hline azote total $(\mathrm{g} / \mathrm{j})$ & 190 & 190 & 190 & 258 & 307 & 307 & 401 & 423 & 423 \\
\hline azote dégradable $(\mathrm{g} / \mathrm{j})$ & 135 & 135 & 135 & 176 & 210 & 210 & 273 & 294 & 294 \\
\hline $\begin{array}{l}\text { Surfaces }\left(\times 10^{-3}\right) \text { des } 2 \\
\text { pics de } \mathrm{NH}_{3}: \\
\text { observées }(\mathrm{mg} \cdot \min / 1)\end{array}$ & 54 & 67 & 74 & 40 & 75 & 87 & 146 & 96 & 157 \\
\hline attendues $(\mathrm{mg} \cdot \mathrm{min} / \mathrm{l})$ (a) & $\begin{array}{l}62 \\
67\end{array}$ & $\begin{array}{l}67 \\
67\end{array}$ & $\begin{array}{l}68 \\
67\end{array}$ & $\begin{array}{l}84 \\
92\end{array}$ & $\begin{array}{c}84 \\
109\end{array}$ & $\begin{array}{c}90 \\
109\end{array}$ & $\begin{array}{l}113 \\
142\end{array}$ & $\begin{array}{l}119 \\
150\end{array}$ & $\begin{array}{l}118 \\
150\end{array}$ \\
\hline $\begin{array}{l}\text { Teneurs journalières en } \mathrm{NH}_{3} \\
(\mathrm{mg} / \mathrm{l})\end{array}$ & 50,8 & 63,6 & 63,1 & 62,1 & 68,3 & 99,7 & 138,2 & 90,6 & 153,4 \\
\hline $\begin{array}{l}\text { Durées journalières des } \\
\text { teneurs en } \\
\mathrm{NH}_{3}<50 \mathrm{mg} / \mathrm{l}(\mathrm{min})\end{array}$ & 930 & 645 & 945 & 350 & 672 & 234 & 0 & 180 & 0 \\
\hline $\mathrm{NH}_{3}>100 \mathrm{mg} / \mathrm{l}(\mathrm{min})$ & 192 & 279 & 333 & 250 & 273 & 570 & 1152 & 528 & 1206 \\
\hline
\end{tabular}

(*) Digestibilité mesurée seulement sur 3 jours consécutifs.

Remerciements. - Travail réalisé sous les auspices de I'I.R.S.I.A., rue de Crayer 6, Bruxelles.

Godeau J.-M., Gillet Y., De Dryer G., 1986. Ann. Méd. vét., 130, 521-526.

Pisulewski P. M., Okorie A. U., Buttery J. P., Haresign W., Lewis D., 1981 J. Sci. Food Agric., 32. 759-766. 